\title{
Adaptive Control for Quadrotors with Uncertain and Faulty Actuators $\star, \star \star$
}

\author{
Qi $\mathrm{Han}^{a}$, Xiangbin Liu ${ }^{b, *}$, Zhitao $\mathrm{Liu}^{a}$ and Hongye $\mathrm{Su}^{a}$ \\ ${ }^{a}$ Institute of Cyber-System and Control, Zhejiang University, Hangzhou, China \\ ${ }^{b}$ School of Electronic Information Engineering, Beijing Jiaotong University, Beijing, China
}

\section{ARTICLE INFO}

\section{Keywords:}

quadrotor

adaptive control

loss of effectiveness

immersion and invariance manifold

\begin{abstract}
A B S T R ACT
Two adaptive control strategies are presented in this paper for two types of quadrotors to cope with potential uncertainties and faults in the actuator system. The four actuators of the considered quadrotors are distinct and suffered from loss of effectiveness (LOE) as well. To accomandate unknown actuator parameters, a filter-based immersion and invariance (I\&I) adaptive controllers are designed for attitude and altitude subsystems, respectively, and an integral backstepping controller is developed for the horizontal subsystem to achieve null steady-state error. Both simulation and experiment results are carried out to illustrate the effectiveness of trajectory tracking performance and fault-tolerant accomondation ability of the proposed control schemes.
\end{abstract}

\section{Introduction}

Quadrotors have received much attention in both military and civilian fields due to mechanical simplicity, low development/maintenance cost and high hovering precision [1]. However, the characteristics of underactuation, nonlinearity, strong coupling and uncertainty of quadrotors bring huge challenges to design the control laws.

The earlier results of quadrotor control are largely based on their physical parameters. In [2, 3, 4], backstepping controllers are designed to stabilize quadrotor system, and in [5, 6], controllers are designed based on the compensation of the Coriolis and gyroscopic torques. To handle the uncertainties in the quadrotor system such as mass, moment of inertia, etc., adaptive control methods are favored by many researchers. In [7], adaptive backstepping method is utilized to estimate the unknown mass, and in [8], an adaptive control scheme is presented to deal with external disturbances and varying center of mass (CoM). To improve transient behaviour, researchers in $[9,10,11]$ utilize I\&I adaptive method which provides a freedom via an additional proportion term to shape the estimation error manifolds, and I\&I adaptive laws are constructed to estimate unknown parameters such as mass and aerodynamic damping coefficients.

With the development of fault-tolerant control (FTC) theory, many researchers are paying more attentions to FTC for quadrotors. It is reported that loss of effectiveness (LOE) fault of actuators occurs frequently in a quadrotor system $[12,13,14]$. In [12], an I\&I observer is constructed to deal with partial LOE (PLOE) of a quadrotor, and it is combined with a sliding mode controller to stabilize the attitude system. In [13], a finite-time fault-tolerant trajectory tracking controller is designed for the quadrotor system against external disturbances, parametric uncertainties and actuator faults without fault diagnosis mechanism. In [14], a systematic design of fault detection, isolation and accommodation is presented for PLOE quadrotor actuator faults using nonlinear adaptive techniques; and a nonlinear robust adaptive fault-tolerant method is developed for altitude and attitude to accommodate actuator faults without the need of a fault diagnosis mechanism in [15]. In [16], fault-tolerant formation control problem is investigated for multi-quadrotor systems based on decentralised state observers and adaptive fault estimators. In [17], an adaptive compensation control scheme is developed via disturbance observer and quantum information technology for the quadrotor, and a reconfiguration control scheme is proposed in [18] for a quadrotor helicopter subject to LOE via simple adaptive control and quantum logic. In [19], a disturbance observer-based adaptive sliding mode control (SMC) strategy for a quadrotor helicopter subject to multiple actuator faults, parametric uncertainties and external disturbances is developed. Intelligent control methods are also investigated by researchers for quadrotor control problem, where in [20], an adaptive PID controller is proposed for a quadrotor system in the presence of actuator faults using the fuzzy inference scheme to tune the controller gains online; and in [21], an indirect neural network (NN)-based adaptive fault-tolerant control scheme is developed, which only involves one lumped parameter adaptation thus is more affordable for onboard implementation.

\footnotetext{
${ }^{\star}$ National Natural Science Foundation of China, Grant/Award Number:61673050. $\operatorname{ORCID}(\mathrm{s})$ :
} 
An extreme situation is considered in [22], where an emergency fault-tolerant controller is developed for quadrotors suffering from a total loss of one rotor.

In this paper, we focus on dealing with the uncertainty in the quadrotor actuator system in controller design. In contrast with a number of existing literature, the parameters of four actuators of the quadrotor have different values. It is due to the fact that, on the one hand, inevitable errors often occur during the manufacturing process and, on the other hand, parameter variation are caused due to long-term operation of the devices. Furthermore, a sudden change in parameters caused by propeller and/or motor damage can also be considered as a special case of actuator uncertainties. To deal with those uncertainties, we take the advantage of I\&I method to design adaptive schemes to compensate them. In the first control scheme, by defining intermediate control inputs, controllers based on I\&I adaptive method with lowpass filters are designed for the attitude and altitude subsystems. However, it is shown that the designed scheme involves self-loops, i.e., algebraic loop, since each control input contains one another. To overcome this problem, we develop the second scheme using gain matrix decomposition technique since the input parameter matrix can be decomposed to several sub-matrices which contain a unity upper triangular matrix. For the horizontal subsystem, a non-adaptive controller is designed, which is combined with the integral term of the tracking error to improve the performance. Finally, we present numerical simulation and experiment to validate the proposed control law. The contribution of this paper are given as follows,

1. Two of the most common configurations of quadrotors are considered, i.e., "+"-type and " $\times$ ”-type. Numerical simulations are carried out on the former type, while experiments are implemented on the latter one.

2. The designed adaptive controller for the corresponding quadrotor is robust to actuator parameter perturbations as well as LOE actuator faults.

3. Sufficicent simulation and experiment results are given to show the superiority performance of the proposed controller compared to the classical adaptive controller.

This paper is organized as follows. Section 2 presents the quadrotor model and problem formulation. In Section 3 , control designs for attitude, altitude and horizontal subsystems are presented respectively, and the stabilities of the closed-loop systems are analyzed. An improved design for attitude and altitude is given in 4 with several notable remarks. A simulated example is provided to show the effectiveness of the proposed approach in Section 5, and experimental results under various situations are carried out in Section 6. Finally, Section 7 draws a conclusion of this study.

\section{Quadrotor Model and Problem Formulation}

We consider two of the most common configurations of quadrotors, namely "+"-type and " $\times$ "-type, whose sketches are shown in the left and right side of Fig. 1, respectively. To describe the motion of quadrotor body, two reference frames are defined as earth inertial reference frame and body-fixed reference frame denoted by $\Sigma_{e}=\left\{x_{e}, y_{e}, z_{e}\right\}$ and $\sum_{b}=\left\{x_{b}, y_{b}, z_{b}\right\}$, respectively. The position and attitude of the quadrotor with respect to $\sum_{e}$ are represented by $[x, y, z]^{T}$ and $[\phi, \theta, \psi]^{T}$, where $\phi, \theta$ and $\psi$ denote roll angle, pitch angle and yaw angle, respectively.

For the two types of quadrotors, they have the same quadrotor body model given as,

$$
\left\{\begin{array}{l}
\ddot{\phi}=\frac{J_{y}-J_{z}}{J_{x}} \dot{\theta} \dot{\psi}-\frac{J_{r}}{J_{x}} \Omega_{R} \dot{\theta}+\frac{\tau_{1}}{J_{x}} \\
\ddot{\theta}=\frac{J_{z}-J_{x}}{J_{y}} \dot{\phi} \dot{\psi}+\frac{J_{r}}{J_{y}} \Omega_{R} \dot{\phi}+\frac{\tau_{2}}{J_{y}} \\
\ddot{\psi}=\frac{J_{x}-J_{y}}{J_{z}} \dot{\phi} \dot{\theta}+\frac{\tau_{3}}{J_{z}} \\
\ddot{z}=-g+(\cos \phi \cos \theta) \frac{f}{m} \\
\ddot{x}=(\cos \phi \sin \theta \cos \psi+\sin \phi \sin \psi) \frac{f}{m} \\
\ddot{y}=(\cos \phi \sin \theta \sin \psi-\sin \phi \cos \psi) \frac{f}{m}
\end{array}\right.
$$




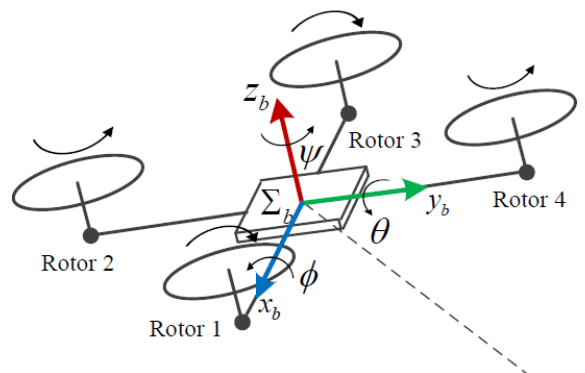

a) “+” type
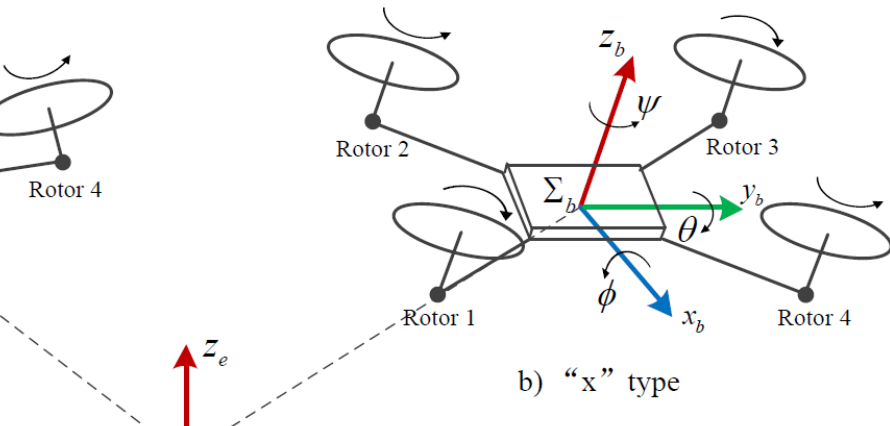

b) “ $\mathrm{x}$ ” type

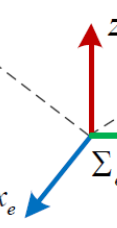

Figure 1: The quadrotor sketches of "+" type and " $x$ " type.

where $J_{x}, J_{y}$ and $J_{z}$ are the moments of inertia along the three body axes; $J_{r}$ is the inertia of the rotor; $\Omega_{R}=\omega_{1}-$ $\omega_{2}+\omega_{3}-\omega_{4}$ with $\omega_{i}, i=1, \cdots, 4$ being the rotor speed; $g$ denotes the gravitational acceleration; $m$ is the mass of quadrotor ; $f, \tau_{1}, \tau_{2}$ and $\tau_{3}$ are control force and torques, respectively.

To faciliate controller design, let $x_{11}=\phi, x_{21}=\theta, x_{31}=\psi, x_{41}=z, x_{51}=x$ and $x_{61}=y$. Each subsystem of the quadrotor model (1) can be expressed in state space form as the following second-order system,

$$
\begin{aligned}
& \dot{x}_{i 1}=x_{i 2} \\
& \dot{x}_{i 2}=f_{i}+g_{i} U_{i}, \quad i=1, \ldots, 6
\end{aligned}
$$

where $f_{i}, g_{i}$ are given as

$$
\begin{aligned}
& f_{1}=\frac{J_{y}-J_{z}}{J_{x}} x_{4} x_{6}-\frac{J_{r}}{J_{x}} \Omega_{r} x_{4}, \quad f_{2}=\frac{J_{z}-J_{x}}{J_{y}} x_{2} x_{6}+\frac{J_{r}}{J_{y}} \Omega_{r} x_{2}, \quad f_{3}=\frac{J_{x}-J_{y}}{J_{z}} x_{2} x_{4}, \quad f_{4}=-g, \\
& f_{5}=f_{6}=0, \quad g_{1}=\frac{1}{J_{x}}, \quad g_{2}=\frac{1}{J_{y}}, \quad g_{3}=\frac{1}{J_{z}}, \quad g_{4}=\frac{\cos x_{1} \cos x_{3}}{m}, \quad g_{5}=g_{6}=\frac{U_{4}}{m} .
\end{aligned}
$$

The six control inputs of subsystems (2) contain four actual inputs and two virtual inputs, i.e., $U_{1}=\tau_{1}, U_{2}=\tau_{2}$, $U_{3}=\tau_{3}$ and $U_{4}=f$ are the actual control inputs; $U_{5}$ and $U_{6}$ are the virtual inputs given as follows,

$$
\begin{aligned}
& U_{5}=\left(\cos x_{1} \sin x_{3} \cos x_{5}+\sin x_{1} \sin x_{5}\right) \\
& U_{6}=\left(\cos x_{1} \sin x_{3} \sin x_{5}-\sin x_{1} \cos x_{5}\right)
\end{aligned}
$$

which are used to obtain the desired roll and pitch angles.

The actual control inputs $U_{i}, i=1, \ldots, 4$ are generated by propellers, which have different formulations for " + "type and " $\times$ "-type respectively. Let actual control inputs of the two types of quadrotor denote by $U_{i}^{+}$and $U_{i}^{\times}$, respectively. For $U_{i}^{+}$, its expression with respect to the propeller speed $\omega_{i}$ is shown as,

$$
\begin{aligned}
& U_{1}^{+}=-c_{t 2} l \omega_{2}^{2}+c_{t 4} l \omega_{4}^{2} \\
& U_{2}^{+}=-c_{t 1} l \omega_{1}^{2}+c_{t 3} l \omega_{3}^{2} \\
& U_{3}^{+}=c_{d 1} \omega_{1}^{2}-c_{d 2} \omega_{2}^{2}+c_{d 3} \omega_{3}^{2}-c_{d 4} \omega_{4}^{2} \\
& U_{4}^{+}=c_{t 1} \omega_{1}^{2}+c_{t 2} \omega_{2}^{2}+c_{t 3} \omega_{3}^{2}+c_{t 4} \omega_{4}^{2} .
\end{aligned}
$$

where $c_{t i}>0$ and $c_{d i}>0, i=1, \ldots, 4$ are the thrust factor and the drag factor of each propeller, respectively, and $l$ is 
the quadrotor arm length. The expression of $U_{i}^{\times}, i=1, \ldots, 4$ is given as

$$
\begin{aligned}
& U_{1}^{\times}=\frac{\sqrt{2}}{2}\left(-c_{t 1} l \omega_{1}^{2}-c_{t 2} l \omega_{2}^{2}+c_{t 3} l \omega_{3}^{2}+c_{t 4} l \omega_{4}^{2}\right) \\
& U_{2}^{\times}=\frac{\sqrt{2}}{2}\left(-c_{t 1} l \omega_{1}^{2}+c_{t 2} l \omega_{2}^{2}+c_{t 3} l \omega_{3}^{2}-c_{t 4} l \omega_{4}^{2}\right) \\
& U_{3}^{\times}=c_{d 1} \omega_{1}^{2}-c_{d 2} \omega_{2}^{2}+c_{d 3} \omega_{3}^{2}-c_{d 4} \omega_{4}^{2} \\
& U_{4}^{\times}=c_{t 1} \omega_{1}^{2}+c_{t 2} \omega_{2}^{2}+c_{t 3} \omega_{3}^{2}+c_{t 4} \omega_{4}^{2} .
\end{aligned}
$$

The four rotors of a quadrotor are all driven by corresponding direct current motors. The rotor speed $\omega_{i}$ is considered to be proportional to the motor voltage input $u_{i}$,

$$
\omega_{i}=K_{m i} u_{i}, \quad i=1, \cdots, 4
$$

with $K_{m i}>0$ the gain coefficient.

Remark 1. Equations (4) and (5) present the relationship between control inputs and rotor speeds of considered two types subject to uncertain parameters. In fact, these equations can also represent the case when actuators are suffering from LOEs. Take "+"-type for example, for the nominal condition, i.e., the parameters of four rotors are identical, then (4) can be expressed as

$$
\begin{aligned}
& U_{1}^{+}=-c_{t} l \omega_{2, a}^{2}+c_{t} l \omega_{4, a}^{2} \\
& U_{2}^{+}=-c_{t} l \omega_{1, a}^{2}+c_{t} l \omega_{3, a}^{2} \\
& U_{3}^{+}=c_{d} \omega_{1, a}^{2}-c_{d} \omega_{2, a}^{2}+c_{d} \omega_{3, a}^{2}-c_{d} \omega_{4, a}^{2} \\
& U_{4}^{+}=c_{t} \omega_{1, a}^{2}+c_{t} \omega_{2, a}^{2}+c_{t} \omega_{3, a}^{2}+c_{t} \omega_{4, a}^{2} .
\end{aligned}
$$

where $\omega_{i, a}$ is the rotor speed under the following actuator fault,

$$
\omega_{i, a}=\varrho_{i} \omega_{i}
$$

with $\varrho_{i} \in(\varrho, 1]$ the LOE factor, and $\varrho>0$ is a lower bound needed to maintain the controllability of the system $[14,15]$. Assume the LOE $\varrho_{1}<1$ occurs to the 1 st rotor while other rotors stay healthy, i.e., $\varrho_{2}=\varrho_{3}=\varrho_{4}=1$, then substitute (8) into (7) yields

$$
\begin{aligned}
& U_{1}^{+}=-c_{t} l \omega_{2}^{2}+c_{t} l \omega_{4}^{2} \\
& U_{2}^{+}=-c_{t 1} l \omega_{1}^{2}+c_{t} l \omega_{3}^{2} \\
& U_{3}^{+}=c_{d 1} \omega_{1}^{2}-c_{d} \omega_{2}^{2}+c_{d} \omega_{3}^{2}-c_{d} \omega_{4}^{2} \\
& U_{4}^{+}=c_{t 1} \omega_{1}^{2}+c_{t} \omega_{2}^{2}+c_{t} \omega_{3}^{2}+c_{t} \omega_{4}^{2} .
\end{aligned}
$$

where $c_{t 1} \triangleq c_{t} \varrho_{1}^{2}$ and $c_{d 1} \triangleq c_{d} \varrho_{1}^{2}$. From this example we see that (4) and (5) can represent the case when all rotors suffer from LOEs.

To facilitate the control design, the following assumptions are made.

Assumption 1. The reference signals and their 2th-order derivatives are available and bounded for the subsystems in (2).

Assumption 2. All state variables are available for controller design.

Assumption 3. The roll angle and pitch angle operate within small ranges.

Remark 2. Assumption 3 is reasonable since it can be guaranteed by a successfully designed controller. Furthermore, it simplifies the model description and the controller design, e.g., the term $g_{4}$ in (2) becomes $g_{4} \approx \frac{1}{m}$ which is constant.

The control objective is to design adaptive controllers for the two types of quadrotors subject to uncertain and faulty actuators, and guarantees that the plant can track the reference given by users. Through out this paper, for simplicity concern, we only make a distinction between the variable names related to the actuator system, since the body dynamics of the two types of quadrotors are the same. 


\section{Adaptive Control Design}

\subsection{Extract actuator parameters}

Observing (4) and (5), where the control inputs $U^{+}$and $U^{\times}$contain unknown and distinct factors. In addition to that, from (6) the gain parameter of each rotor speed to the voltage input is also unknown. Hence, we need to extract actuator parameters from the original control inputs $U^{+}$and $U^{\times}$to facilitate the control design.

For “+”-type quadrotor, substituting (6) into (4),

$$
\begin{aligned}
& U_{1}^{+}=-\bar{\vartheta}_{11}^{+} u_{2}^{2}+\bar{\vartheta}_{12}^{+} u_{4}^{2} \\
& U_{2}^{+}=-\bar{\vartheta}_{21}^{+} u_{1}^{2}+\bar{\vartheta}_{22}^{+} u_{3}^{2} \\
& U_{3}^{+}=\bar{\vartheta}_{31}^{+} u_{1}^{2}-\bar{\vartheta}_{32}^{+} u_{2}^{2}+\bar{\vartheta}_{33}^{+} u_{3}^{2}-\bar{\vartheta}_{34}^{+} u_{4}^{2} \\
& U_{4}^{+}=\bar{\vartheta}_{41}^{+} u_{1}^{2}+\bar{\vartheta}_{42}^{+} u_{2}^{2}+\bar{\vartheta}_{43}^{+} u_{3}^{2}+\bar{\vartheta}_{44}^{+} u_{4}^{2}
\end{aligned}
$$

where $\bar{\vartheta}_{11}^{+}=c_{t 2} l K_{m 2}^{2}, \bar{\vartheta}_{12}^{+}=c_{t 4} l K_{m 4}^{2}, \bar{\vartheta}_{21}^{+}=c_{t 1} l K_{m 1}^{2}, \bar{\vartheta}_{22}^{+}=c_{t 3} l K_{m 3}^{2}, \bar{\vartheta}_{3 i}^{+}=c_{d i} K_{m i}^{2}$ and $\bar{\vartheta}_{4 i}^{+}=c_{t i} K_{m i}^{2}$.

Define the intermediate variables $v_{i}^{+}, i=1, \cdots, 4$ as

$$
\begin{aligned}
& v_{1}^{+}=-u_{2}^{2}+u_{4}^{2} \\
& v_{2}^{+}=-u_{1}^{2}+u_{3}^{2} \\
& v_{3}^{+}=u_{1}^{2}-u_{2}^{2}+u_{3}^{2}-u_{4}^{2} \\
& v_{4}^{+}=u_{1}^{2}+u_{2}^{2}+u_{3}^{2}+u_{4}^{2}
\end{aligned}
$$

Using (8), then (7) can be rewritten as,

$$
\begin{aligned}
& U_{1}^{+}=\vartheta_{11}^{+} v_{1}^{+}+\vartheta_{12}^{+}\left(v_{3}^{+}-v_{4}^{+}\right) \\
& U_{2}^{+}=\vartheta_{21}^{+}\left(v_{3}^{+}+v_{4}^{+}\right)+\vartheta_{22}^{+} v_{2}^{+} \\
& U_{3}^{+}=\vartheta_{31}^{+} v_{1}^{+}+\vartheta_{32}^{+} v_{2}^{+}+\vartheta_{33}^{+} v_{3}^{+}+\vartheta_{34}^{+} v_{4}^{+} \\
& U_{4}^{+}=\vartheta_{41}^{+} v_{1}^{+}+\vartheta_{42}^{+} v_{2}^{+}+\vartheta_{43}^{+} v_{3}^{+}+\vartheta_{44}^{+} v_{4}^{+}
\end{aligned}
$$

where

$$
\begin{aligned}
& \vartheta_{11}^{+}=\frac{\bar{\vartheta}_{11}^{+}+\bar{\vartheta}_{12}^{+}}{2}, \quad \vartheta_{12}^{+}=\frac{\bar{\vartheta}_{11}^{+}-\bar{\vartheta}_{12}^{+}}{4}, \quad \vartheta_{21}^{+}=\frac{-\bar{\vartheta}_{21}^{+}+\bar{\vartheta}_{22}^{+}}{4}, \quad \vartheta_{22}^{+}=\frac{\bar{\vartheta}_{21}^{+}+\bar{\vartheta}_{22}^{+}}{2}, \\
& \vartheta_{31}^{+}=\frac{\bar{\vartheta}_{32}^{+}-\bar{\vartheta}_{34}^{+}}{2}, \quad \vartheta_{32}^{+}=\frac{-\bar{\vartheta}_{31}^{+}+\bar{\vartheta}_{33}^{+}}{2}, \quad \vartheta_{33}^{+}=\frac{\sum_{i=1}^{4} \bar{\vartheta}_{3 i}^{+}}{4}, \quad \vartheta_{34}^{+}=\frac{\sum_{i=1}^{4}(-1)^{i+1} \bar{\vartheta}_{3 i}^{+}}{4}, \\
& \vartheta_{41}^{+}=\frac{-\bar{\vartheta}_{42}^{+}+\bar{\vartheta}_{44}^{+}}{2}, \quad \vartheta_{42}^{+}=\frac{-\bar{\vartheta}_{41}^{+}+\bar{\vartheta}_{43}^{+}}{2}, \quad \vartheta_{43}^{+}=\frac{\sum_{i=1}^{4}(-1)^{i+1} \bar{\vartheta}_{4 i}^{+}}{4}, \quad \vartheta_{44}^{+}=\frac{\sum_{i=1}^{4} \bar{\vartheta}_{4 i}^{+}}{4}
\end{aligned}
$$

are unknown parameters.

For “ $\times$ ”-type quadrotor, substituting (6) into (5),

$$
\begin{aligned}
& U_{1}^{\times}=-\bar{\vartheta}_{11}^{\times} u_{1}^{2}-\bar{\vartheta}_{12}^{\times} u_{2}^{2}+\bar{\vartheta}_{13}^{\times} u_{3}^{2}+\bar{\vartheta}_{14}^{\times} u_{4}^{2} \\
& U_{2}^{\times}=-\bar{\vartheta}_{21}^{\times} u_{1}^{2}+\bar{\vartheta}_{22}^{\times} u_{2}^{2}+\bar{\vartheta}_{23}^{\times} u_{3}^{2}-\bar{\vartheta}_{24}^{\times} u_{4}^{2} \\
& U_{3}^{\times}=\bar{\vartheta}_{31}^{\times} u_{1}^{2}-\bar{\vartheta}_{32}^{\times} u_{2}^{2}+\bar{\vartheta}_{33}^{\times} u_{3}^{2}-\bar{\vartheta}_{34}^{\times} u_{4}^{2} \\
& U_{4}^{\times}=\bar{\vartheta}_{41}^{\times} u_{1}^{2}+\bar{\vartheta}_{42}^{\times} u_{2}^{2}+\bar{\vartheta}_{43}^{\times} u_{3}^{2}+\bar{\vartheta}_{44}^{\times} u_{4}^{2}
\end{aligned}
$$

where $\bar{\vartheta}_{1 i}^{\times}=\bar{\vartheta}_{2 i}^{\times}=\sqrt{2} c_{t i} l K_{m i}^{2} / 2, \bar{\vartheta}_{3 i}^{\times}=c_{d i} K_{m i}^{2}$ and $\bar{\vartheta}_{4 i}^{\times}=c_{t i} K_{m i}^{2}$. 
Define intermediate variables $v_{i}^{\times}, i=1, \cdots, 4$, as

$$
\begin{aligned}
& v_{1}^{\times}=-u_{1}^{2}-u_{2}^{2}+u_{3}^{2}+u_{4}^{2} \\
& v_{2}^{\times}=-u_{1}^{2}+u_{2}^{2}+u_{3}^{2}-u_{4}^{2} \\
& v_{3}^{\times}=u_{1}^{2}-u_{2}^{2}+u_{3}^{2}-u_{4}^{2} \\
& v_{4}^{\times}=u_{1}^{2}+u_{2}^{2}+u_{3}^{2}+u_{4}^{2}
\end{aligned}
$$

then (10) can be rewritten using (11),

$$
\begin{aligned}
& U_{1}^{\times}=\vartheta_{11}^{\times} v_{1}^{\times}+\vartheta_{12}^{\times} v_{2}^{\times}+\vartheta_{13}^{\times} v_{3}^{\times}+\vartheta_{14}^{\times} v_{4}^{\times} \\
& U_{2}^{\times}=\vartheta_{21}^{\times} v_{1}^{\times}+\vartheta_{22}^{\times} v_{2}^{\times}+\vartheta_{23}^{\times} v_{3}^{\times}+\vartheta_{24}^{\times} v_{4}^{\times} \\
& U_{3}^{\times}=\vartheta_{31}^{\times} v_{1}^{\times}+\vartheta_{32}^{\times} v_{2}^{\times}+\vartheta_{33}^{\times} v_{3}^{\times}+\vartheta_{34}^{\times} v_{4}^{\times} \\
& U_{4}^{\times}=\vartheta_{41}^{\times} v_{1}^{\times}+\vartheta_{42}^{\times} v_{2}^{\times}+\vartheta_{43}^{\times} v_{3}^{\times}+\vartheta_{44}^{\times} v_{4}^{\times}
\end{aligned}
$$

where

$$
\begin{aligned}
& \vartheta_{11}^{\times}=\frac{\sum_{i=1}^{4} \bar{\vartheta}_{1 i}^{\times}}{4}, \quad \vartheta_{12}^{\times}=\frac{\sum_{i=1}^{4}(-1)^{i+1} \bar{\vartheta}_{1 i}^{\times}}{4}, \quad \vartheta_{13}^{\times}=\frac{-\bar{\vartheta}_{11}^{\times}+\bar{\vartheta}_{12}^{\times}+\bar{\vartheta}_{13}^{\times}-\bar{\vartheta}_{14}^{\times}}{4}, \quad \vartheta_{14}^{\times}=\frac{-\bar{\vartheta}_{11}^{\times}-\bar{\vartheta}_{12}^{\times}+\bar{\vartheta}_{13}^{\times}+\bar{\vartheta}_{14}^{\times}}{4}, \\
& \vartheta_{21}^{\times}=\frac{\sum_{i=1}^{4}(-1)^{i+1} \bar{\vartheta}_{2 i}^{\times}}{4}, \quad \vartheta_{22}^{\times}=\frac{\sum_{i=1}^{4} \bar{\vartheta}_{2 i}^{\times}}{4}, \quad \vartheta_{23}^{\times}=\frac{-\bar{\vartheta}_{21}^{\times}-\bar{\vartheta}_{22}^{\times}+\bar{\vartheta}_{23}^{\times}+\bar{\vartheta}_{24}^{\times}}{4}, \quad \vartheta_{24}^{\times}=\frac{-\bar{\vartheta}_{21}^{\times}+\bar{\vartheta}_{22}^{\times}+\bar{\vartheta}_{23}^{\times}-\bar{\vartheta}_{24}^{\times}}{4}, \\
& \vartheta_{31}^{\times}=\frac{-\bar{\vartheta}_{31}^{\times}+\bar{\vartheta}_{32}^{\times}+\bar{\vartheta}_{33}^{\times}-\bar{\vartheta}_{34}^{\times}}{4}, \quad \vartheta_{32}^{\times}=\frac{-\bar{\vartheta}_{31}^{\times}-\bar{\vartheta}_{32}^{\times}+\bar{\vartheta}_{33}^{\times}+\bar{\vartheta}_{34}^{\times}}{4}, \quad \vartheta_{33}^{\times}=\frac{\sum_{i=1}^{4} \bar{\vartheta}_{3 i}^{\times}}{4}, \quad \vartheta_{34}^{\times}=\frac{\sum_{i=1}^{4}(-1)^{i+1} \bar{\vartheta}_{3 i}^{\times}}{4}, \\
& \vartheta_{41}^{\times}=\frac{-\bar{\vartheta}_{41}^{\times}-\bar{\vartheta}_{42}^{\times}+\bar{\vartheta}_{43}^{\times}+\bar{\vartheta}_{44}^{\times}}{4}, \quad \vartheta_{42}^{\times}=\frac{-\bar{\vartheta}_{41}^{\times}+\bar{\vartheta}_{42}^{\times}+\bar{\vartheta}_{43}^{\times}-\bar{\vartheta}_{44}^{\times}}{4}, \quad \vartheta_{43}^{\times}=\frac{\sum_{i=1}^{4}(-1)^{i+1} \bar{\vartheta}_{4 i}^{\times}}{4}, \quad \vartheta_{44}^{\times}=\frac{\sum_{i=1}^{4} \bar{\vartheta}_{4 i}^{\times}}{4}
\end{aligned}
$$

are unknown parameters.

To unify the expressions of "+"-type and " $x$ "-type, the attitude and altitude subsystems in system (2) is rewritten as

$$
\begin{aligned}
& \dot{x}_{i 1}=x_{i 2} \\
& \dot{x}_{i 2}=f_{i}+g_{i}\left(\vartheta_{i i}^{*} v_{i}^{*}+\varphi_{i}^{* T} \vartheta_{i}^{*}\right), \quad i=1, \cdots, 4
\end{aligned}
$$

where the superscript $*$ denotes + or $\times$, and

$$
\begin{aligned}
& \varphi_{1}^{+}=v_{3}^{+}-v_{4}^{+}, \quad \varphi_{2}^{+}=v_{3}^{+}+v_{4}^{+}, \quad \varphi_{3}^{+}=\left[v_{1}^{+}, v_{2}^{+}, v_{4}^{+}\right]^{T}, \quad \varphi_{4}^{+}=\left[v_{1}^{+}, v_{2}^{+}, v_{3}^{+}\right]^{T}, \\
& \vartheta_{1}^{+}=\vartheta_{12}^{+}, \quad \vartheta_{2}^{+}=\vartheta_{21}^{+}, \quad \vartheta_{3}^{+}=\left[\vartheta_{31}^{+}, \vartheta_{32}^{+}, \vartheta_{34}^{+}\right]^{T}, \quad \vartheta_{4}^{+}=\left[\vartheta_{41}^{+}, \vartheta_{42}^{+}, \vartheta_{43}^{+}\right]^{T}, \\
& \varphi_{1}^{\times}=\left[v_{2}^{\times}, v_{3}^{\times}, v_{4}^{\times}\right]^{T}, \quad \varphi_{2}^{\times}=\left[v_{1}^{\times}, v_{3}^{\times}, v_{4}^{\times}\right]^{T} \quad \varphi_{3}^{\times}=\left[v_{1}^{\times}, v_{2}^{\times}, v_{4}^{\times}\right]^{T}, \quad \varphi_{4}^{\times}=\left[v_{1}^{\times}, v_{2}^{\times}, v_{3}^{\times}\right]^{T} \\
& \vartheta_{1}^{\times}=\left[\vartheta_{12}^{\times}, \vartheta_{13}^{\times}, \vartheta_{14}^{\times}\right]^{T}, \quad \vartheta_{2}^{\times}=\left[\vartheta_{21}^{\times}, \vartheta_{23}^{\times}, \vartheta_{24}^{\times}\right]^{T}, \quad \vartheta_{3}^{\times}=\left[\vartheta_{31}^{\times}, \vartheta_{32}^{\times}, \vartheta_{34}^{\times}\right]^{T}, \quad \vartheta_{4}^{\times}=\left[\vartheta_{41}^{\times}, \vartheta_{42}^{\times}, \vartheta_{43}^{\times}\right]^{T} .
\end{aligned}
$$

In what follows, $v_{i}^{*}$ is the control input to be designed. Obviously, after determining $v_{i}^{*}$, one can obtain the desired voltage inputs $u_{i}$ according to (11) and (14), respectively.

\subsection{Adaptive Control Design for Attitude and Altitude Subsystems}

Compared with the classical adaptive method which depends on cancellation of the terms of the estimation error related in the derivative of Lyapunov function, I\&I adaptive approach provides an effective means of shaping the dynamic response of the estimation error via domination [23]. In view of this, we design an adaptive controller based on I\&I adaptive method for attitude and altitude subsystems of the quadrotor. Since solving the partial differential equation (PDE) is needed in I\&I adaptive method, hence, the control design will be presented via the introduction of linear low-pass filters to bypass the solving of PDEs. The filter-based I\&I adaptive control method has been applied in various kinds of systems (see [23, 24, 25] for example). 
To begin with, for attitude and altitude subsystems (16), define the following error variables as ,

$$
z_{i 1}=x_{i 1}-x_{i d}, \quad z_{i 2}=x_{i 2}-\alpha_{i}, \quad i=1, \cdots, 4
$$

where $x_{i d}$ is the reference signal of each subsystem, $\alpha_{i}$ is the virtual control to be designed. The derivative of $z_{i 1}$ is calculated as

$$
\dot{z}_{i 1}=z_{i 2}+\alpha_{i}-\dot{x}_{i d}
$$

$\alpha_{i}$ is designed as

$$
\alpha_{i}=-k_{i 1} z_{i 1}+\dot{x}_{i d}
$$

with design parameter $k_{i 1}>0$, then (18) is written as

$$
\dot{z}_{i 1}=-k_{i 1} z_{i 1}+z_{i 2}
$$

The derivative of $z_{i 2}$ is calculated as

$$
\begin{aligned}
\dot{z}_{i 2} & =\dot{x}_{i 2}-\dot{\alpha}_{i} \\
& =f_{i}+g_{i}\left(\vartheta_{i i}^{*} v_{i}^{*}+\varphi_{i}^{* T} \vartheta_{i}^{*}\right)-\dot{\alpha}_{i} \\
& =g_{i} \vartheta_{i i}^{*} v_{i}^{*}+\bar{\varphi}_{i}^{* T} \bar{\vartheta}_{i}^{*} .
\end{aligned}
$$

where $\bar{\varphi}_{i}^{* T} \bar{\vartheta}_{i}^{*}=g_{i} \varphi_{i}^{* T} \vartheta_{i}^{*}+f_{i}-\dot{\alpha}_{i}$ with $\bar{\varphi}_{i}^{*}$ a known function vector and $\bar{\vartheta}_{i}^{*}$ an unknown constant vector.

Based on the above analysis, the adaptive control law $v_{i}^{*}$ will be specified as the following theorem.

Theorem 1. Define the following filters

$$
\begin{aligned}
\dot{z}_{i 1, f} & =-z_{i 1, f}+z_{i 1} \\
\dot{z}_{i 2, f} & =-z_{i 2, f}+z_{i 2} \\
\dot{\varphi}_{i, f}^{*} & =-\bar{\varphi}_{i, f}^{*}+\bar{\varphi}_{i}^{*} \\
\dot{v}_{i, f}^{*} & =-v_{i, f}^{*}+v_{i}^{*}
\end{aligned}
$$

which yield the following error dynamics,

$$
\begin{aligned}
& \dot{z}_{i 1, f}=-k_{i 1} z_{i 1, f}+z_{i 2, f}+\varepsilon_{1} \\
& \dot{z}_{i 2, f}=g_{i} \vartheta_{i i}^{*} v_{i, f}^{*}+\bar{\varphi}_{i, f}^{* T} \bar{\vartheta}_{i}^{*}+\varepsilon_{2}
\end{aligned}
$$

where $\varepsilon_{1}$ and $\varepsilon_{2}$ are exponentially decaying terms to be specified later. For system (2), the I\&I adaptive controller is designed as,

$$
\begin{aligned}
v_{i, f}^{*} & =-z_{i 1, f}-k_{i 2} z_{i 2, f}-\bar{\varphi}_{i, f}^{* T}\left(\hat{\vartheta}_{i}^{*}+\beta_{i}^{*}\right) \\
\hat{\hat{\vartheta}}_{i}^{*} & =\gamma_{i} \bar{\varphi}_{i, f}^{*}\left(z_{i 1, f}+k_{i 2} z_{i 2, f}\right)-\gamma_{i} z_{i 2, f} \dot{\bar{\varphi}}_{i, f}^{*} \\
\beta_{i}^{*} & =\gamma_{i} \bar{\varphi}_{i, f}^{*} z_{i 2, f}
\end{aligned}
$$

where $k_{i 2}>0, \gamma_{i}>0$ are the design parameters and $\left(\hat{\vartheta}_{i}^{*}+\beta_{i}^{*}\right)$ are the estimate of $\frac{\bar{\vartheta}_{i}^{*}}{g_{i} \vartheta_{i i}^{*}}$. Then, all the signals in the closed-loop system are ultimately bounded and attitude and altitude tracking errors $z_{i 1}$ converge to zero.

PROOF. Define the following variables

$$
\begin{aligned}
& \eta_{i 1}=k_{i 1} z_{i 1, f}-z_{i 2, f}-z_{i 1, f}+z_{i 1} \\
& \eta_{i 2}=-g_{i}\left(\vartheta_{i i}^{*} v_{i, f}^{*}+\bar{\varphi}_{i, f}^{* T} \bar{\vartheta}_{i}^{*}\right)-z_{i 2, f}+z_{i 2}
\end{aligned}
$$


It has $\dot{\eta}_{i 1}=-\eta_{i 1}$ and $\dot{\eta}_{i 2}=-\eta_{i 2}$ from (22)- (27), and $\varepsilon_{1}$ and $\varepsilon_{2}$ are dropped hereafter since they do not affect the validity of the stability analysis[23, 24, 25]. The filtered error system is now represented as

$$
\begin{aligned}
& \dot{z}_{i 1, f}=-k_{i 1} z_{i 1, f}+z_{i 2, f} \\
& \dot{z}_{i 2, f}=g_{i} \vartheta_{i i}^{*} v_{i, f}^{*}+\bar{\varphi}_{i, f}^{* T} \bar{\vartheta}_{i}^{*}
\end{aligned}
$$

Substituting control law (28) into (33) yields

$$
\dot{z}_{i 2, f}=g_{i} \vartheta_{i i}^{*}\left(-z_{i 1, f}-k_{i 2} z_{i 2, f}-\bar{\varphi}_{i, f}^{* T} \zeta_{i}^{*}\right)
$$

with

$$
\zeta_{i}^{*}=\hat{\vartheta}_{i}^{*}-\frac{\bar{\vartheta}_{i}^{*}}{g_{i} \vartheta_{i i}^{*}}+\beta_{i}^{*}
$$

defined as the off-the-manifold, whose derivative can be obtained by invoking (29) and (30),

$$
\dot{\zeta}_{i}^{*}=-\gamma_{i} \bar{\varphi}_{i, f}^{*} \bar{\varphi}_{i, f}^{* T} \zeta_{i}^{*}
$$

Finally, the stability of whole closed-loop system can be verified by choosing the Lyapunov function

$$
V_{1}^{*}=\frac{1}{2} \sum_{i=1}^{4}\left(z_{i 1, f}^{2}+\frac{1}{g_{i} \vartheta_{i i}^{*}} z_{i 2, f}^{2}+\frac{1}{k_{i 2}} \zeta_{i}^{* T} \gamma_{i}^{-1} \zeta_{i}^{*}\right)
$$

whose time derivative along the solutions of (32), (34) and (36) is given by

$$
\begin{aligned}
\dot{V}_{1}^{*} & =\sum_{i=1}^{4}\left(z_{i 1, f} \dot{z}_{i 1, f}+\frac{1}{g_{i} \vartheta_{i i}^{*}} z_{i 2, f} \dot{z}_{i 2, f}+\frac{1}{k_{i 2}} \zeta_{i}^{* T} \gamma^{-1} \dot{\zeta}_{i}^{*}\right) \\
& =\sum_{i=1}^{4}\left(-k_{i 1} z_{i 1, f}^{2}-k_{i 2} z_{i 2, f}^{2}-\bar{\varphi}_{i, f}^{* T} \zeta_{i}^{*} z_{i 2, f}-\frac{1}{k_{i 2}}\left(\bar{\varphi}_{i, f}^{* T} \zeta_{i}^{*}\right)^{2}\right) \\
& \leq \sum_{i=1}^{4}\left(-k_{i 1} z_{i 1, f}^{2}-\frac{k_{i 2}}{2} z_{i 2, f}^{2}-\frac{1}{2 k_{i 2}}\left(\bar{\varphi}_{i, f}^{* T} \zeta_{i}^{*}\right)^{2}\right) \\
& \leq 0 .
\end{aligned}
$$

Then it can concluded that $V_{1}^{*}(t) \in \ell_{\infty}$, and thus $z_{i 1, f} \in \ell_{\infty}, z_{i 2, f} \in \ell_{\infty}$ and $\zeta_{i}^{*} \in \ell_{\infty}$. From the definition of $\zeta_{i}^{*}$, the boundedness of $\hat{\vartheta}_{i}^{*}+\beta_{i}^{*}$ is guaranteed. Then $v_{i, f}^{*}$ is bounded and consequently all the closed-loop signals are bounded.

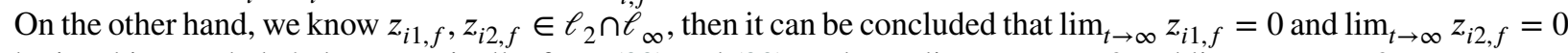
by invoking Barbalat's lemma. Finally, from (22) and (23) we know $\lim _{t \rightarrow \infty} z_{i 1}=0$ and $\lim _{t \rightarrow \infty} z_{i 2}=0$.

Moreover, the actual control $v_{i}^{*}$ should be recovered according to (25) as,

$$
v_{i}^{*}=\dot{v}_{i, f}^{*}+v_{i, f}^{*}
$$

from which we know that the analytical expression of $\dot{v}_{i, f}^{*}$ is available from (22)-(24). However, it can be seen that $\dot{v}_{i, f}^{*}$ contains $\dot{\bar{\varphi}}_{i, f}^{*}$ and thus contains the unfiltered regressors $\bar{\varphi}_{i}^{*}$ by recalling (24). From the definition of $\bar{\varphi}_{i}^{*}$, we can observe that it involves $\varphi_{i}^{*}$, which consists of $v_{i}^{*}$. Thus, it causes a self-loop problem where each control input $v_{i}^{*}$ contains one another. In Section 4, an alternative adaptive design will be presented to address the problem. The design for horizontal control will be presented here first. 


\subsection{Control Design for Horizontal Subsystem}

Rewrite the horizontal subsystem from (2) as

$$
\begin{aligned}
& \dot{x}_{j 1}=x_{j 2} \\
& \dot{x}_{j 2}=\rho U_{j}, j=5,6
\end{aligned}
$$

where $\rho=\frac{U_{4}^{*}}{m}$ is unknown from (4) or (5) and is always positive for dominating the gravity. Define the following error variables

$$
z_{j 1}=e_{j}+k_{0} \int_{0}^{t} e_{j} d t, \quad z_{j 2}=x_{j 2}-\alpha_{j}
$$

where $e_{j}=x_{j 1}-x_{j d}$ is the tracking error, $x_{j d}$ is the reference signal, $k_{0}>0$ is a positive constant, and $\alpha_{j}$ is the virtual control law. Differentiating $z_{j 1}$ gives

$$
\begin{aligned}
\dot{z}_{j 1} & =\dot{e}_{j}+k_{0} e_{j} \\
& =z_{j 2}+\alpha_{j}-\dot{x}_{j d}+k_{0} e_{j}
\end{aligned}
$$

Design $\alpha_{j}$ in (42) as

$$
\alpha_{j}=-k_{j 1} z_{j 1}+\dot{x}_{j d}-k_{0} e_{j}
$$

with $k_{j 1}>0,(42)$ is rewritten as

$$
\dot{z}_{j 1}=-k_{j 1} z_{j 1}+z_{j 2} .
$$

Differentiating $z_{j 2}$ gives

$$
\dot{z}_{j 2}=\rho U_{j}-\dot{\alpha}_{j}
$$

The horizontal control law $U_{j}$ is designed as

$$
U_{j}=-\left(k_{j 2}+\dot{\alpha}_{j}^{2}\right) z_{j 2}
$$

with the control parameter $k_{j 2}>0$. The stability of under control (46) can be checked by Lyapunov function $V_{h}=$ $\frac{z_{51}^{2}}{2}+\frac{z_{61}^{2}}{2 k_{51}}+\frac{z_{52}^{2}}{2}+\frac{z_{62}^{2}}{2 k_{61}}$, whose derivative along the solutions of (44) and (45) is

$$
\dot{V}_{h}=\sum_{j=5}^{6}\left[-k_{j 1} z_{j 1}^{2}+z_{j 1} z_{j 2}-k_{j 1}^{-1} \rho\left(k_{j 2}+\dot{\alpha}_{j}^{2}\right) z_{j 2}^{2}-k_{j 1}^{-1} \dot{\alpha}_{j} z_{j 2}\right]
$$

Using Young's inequality on the cross terms in (47),

$$
\begin{aligned}
z_{j 1} z_{j 2} & \leq \frac{k_{j 1}}{2} z_{j 1}^{2}+\frac{1}{2 k_{j 1}} z_{j 2}^{2} \\
\dot{\alpha}_{j} z_{j 2} & \leq \frac{\rho}{k_{j 1}} \dot{\alpha}_{j}^{2} z_{j 2}^{2}+\lambda_{j}
\end{aligned}
$$

where $\underline{\rho}=\inf \{\rho\}>0$ and $\lambda_{j}=\frac{k_{j 1}}{4 \underline{\rho}},(47)$ becomes

$$
\begin{aligned}
\dot{V}_{h} & \leq \sum_{j=5}^{6}\left(-\frac{1}{2} k_{j 1} z_{j 1}^{2}-\frac{\rho k_{j 2}}{k_{j 1}} z_{j 2}^{2}+\lambda_{j}\right) \\
& \leq-k_{h} V_{h}+\lambda_{h}
\end{aligned}
$$


where $k_{h}=\min \left\{k_{j h}\right\}$ with $k_{j h}=\min \left\{k_{j 1}, 2 \rho k_{j 2}\right\}$ and $\lambda_{h}=\lambda_{5}+\lambda_{6}$. Integrating both side of (48), one has

$$
V_{h}(t) \leq\left(V_{h}(0)-\frac{\lambda_{h}}{k_{h}}\right) \exp \left(-k_{h} t\right)+\frac{\lambda_{h}}{k_{h}} .
$$

Therefore, $V_{h}(t) \in \ell_{\infty}$, and thus $z_{j 1}, z_{j 2} \in \ell_{\infty}$. The bound of horizontal tracking error $z_{j 1}$ can be found as $\sqrt{2 \lambda_{h} / k_{h}}$.

Remark 3. It can be observed that the above horizontal control design includes the integral of the tracking error. The existence of integral action provides an additional design of freedom, and the horizontal tracking accuracy could be improved even when the performance of roll and pitch angle are not good enough.

\section{Adaptive Control Design via Gain Matrix Decomposition}

As illustrated in Section 3, the above adaptive control design for attitude and altitude subsystems involves algebraic loops. In this section, inspired by [26, 27, 28], an adaptive control design is designed to solve self-loop problem using gain matrix decomposition method. After presenting this control scheme, we will also present an alternative design with less filters applied and is easier to implement in practical.

Lemma 1. [26, 27, 28] Define the leading principal minors of a real matrix $G \in \mathbb{R}^{q \times q}$ as $\Delta_{1}, \cdots, \Delta_{q}$. If $\Delta_{1}, \cdots, \Delta_{q}$ are nonzero, then $G$ can be decomposed as

$$
G=S D Q
$$

where $S$ is symmetric positive definite matrix, $D=D_{1} D_{2}$ is diagonal with $D_{1}$ arbitrary positive diagonal and $D_{2}=\operatorname{diag}\left\{\operatorname{sgn}\left(\Delta_{1}\right), \operatorname{sgn}\left(\frac{\Delta_{2}}{\Delta_{1}}\right), \cdots, \operatorname{sgn}\left(\frac{\Delta_{q}}{\Delta_{q-1}}\right)\right\}$, and $Q$ is unity upper triangular.

Define $U^{*}=\left[U_{1}^{*}, U_{2}^{*}, U_{3}^{*}, U_{4}^{*}\right]^{T}, v^{*}=\left[v_{1}^{*}, v_{2}^{*}, v_{3}^{*}, v_{4}^{*}\right]^{T}$, and rewrite (12) and (15) in the matrix form as

$$
\begin{aligned}
U^{+}=G^{+} v^{+}= & {\left[\begin{array}{cccc}
\vartheta_{11}^{+} & 0 & \vartheta_{12}^{+} & -\vartheta_{12}^{+} \\
0 & \vartheta_{22}^{+} & \vartheta_{21}^{+} & \vartheta_{21}^{+} \\
\vartheta_{31}^{+} & \vartheta_{32}^{+} & \vartheta_{33}^{+} & \vartheta_{34}^{+} \\
\vartheta_{41}^{+} & \vartheta_{42}^{+} & \vartheta_{43}^{+} & \vartheta_{44}^{+}
\end{array}\right] v^{+} } \\
U^{\times}=G^{\times} v^{\times} & =\left[\begin{array}{cccc}
\vartheta_{11}^{\times} & \vartheta_{12}^{\times} & \vartheta_{13}^{\times} & \vartheta_{14}^{\times} \\
\vartheta_{21}^{\times} & \vartheta_{22}^{\times} & \vartheta_{23}^{\times} & \vartheta_{24}^{\times} \\
\vartheta_{31}^{\times} & \vartheta_{32}^{\times} & \vartheta_{33}^{\times} & \vartheta_{34}^{\times} \\
\vartheta_{41}^{\times} & \vartheta_{42}^{\times} & \vartheta_{43}^{\times} & \vartheta_{44}^{\times}
\end{array}\right] v^{\times}
\end{aligned}
$$

To utilize Lemma 1, the following assumption is made in addition.

Assumption 4. The leading principal minors of matrix $G^{*}$ are nonzero, and their signs are known.

Using Lemma 1 under Assumption 4, matrix $G^{*}$ can be decomposed as

$$
G^{*}=S^{*} D^{*} Q^{*}
$$

then (12) can be rewritten as

$$
\begin{aligned}
\left(\bar{g} S^{*}\right)^{-1} \dot{z}_{2} & =D^{*} Q^{*} v^{*}+\left(\bar{g} S^{*}\right)^{-1}(f-\dot{\alpha}) \\
& =D^{*}\left[v^{*}-\left(1-Q^{*}\right) v^{*}\right]+\left(\bar{g} S^{*}\right)^{-1}(f-\dot{\alpha}) \\
& =D^{*} v^{*}+\mu \Theta^{*}
\end{aligned}
$$

where $\bar{g}=\operatorname{diag}\left\{g_{1}, g_{2}, g_{3}, g_{4}\right\}, f=\left[f_{1}, f_{2}, f_{3}, f_{4}\right]^{T}, \dot{\alpha}=\left[\dot{\alpha}_{1}, \dot{\alpha}_{2}, \dot{\alpha}_{3}, \dot{\alpha}_{4}\right]^{T}, z_{2}=\left[z_{12}, z_{22}, z_{32}, z_{42}\right]^{T}, v^{*}=\left[v_{1}^{*}, v_{2}^{*}, v_{3}^{*}, v_{4}^{*}\right]^{T}$ and $\mu \Theta^{*}$ denotes

$$
\mu \Theta^{*}=-D^{*}\left(1-Q^{*}\right) v^{*}+\left(\bar{g} S^{*}\right)^{-1}(f-\dot{\alpha})
$$


with $\mu^{*}=\operatorname{diag}\left\{\mu_{1}^{* T}, \mu_{2}^{* T}, \mu_{3}^{* T}, \mu_{4}^{* T}\right\} \in \mathbb{R}^{4 \times p_{i}}, p_{i}>0$ being a known function matrix and $\Theta^{*} \in \mathbb{R}^{p_{i}}$ an unknown parameter vector.

Introducing the following filter

$$
\dot{\mu}_{f}^{*}=-\mu_{f}^{*}+\mu^{*}
$$

with filters given in (22), (23) and (25), the filtered version of (53) is

$$
\left(\bar{g} S^{*}\right)^{-1} \dot{z}_{2, f}=D^{*} v_{f}^{*}+\mu_{f} \Theta^{*}
$$

where $z_{2, f}=\left[z_{12, f}, z_{22, f}, z_{32, f}, z_{42, f}\right]^{T}$ and $v_{f}^{*}=\left[v_{1, f}^{*}, v_{2, f}^{*}, v_{3, f}^{*}, v_{4, f}^{*}\right]^{T}$. The control will be given in the following theorem.

Theorem 2. For system (2), the I\&I adaptive controller is designed as

$$
\begin{aligned}
v_{f}^{*} & =D^{*-1}\left[-z_{1, f}-k_{2} z_{2, f}-\mu_{f}\left(\hat{\Theta}^{*}+\beta_{\Theta}^{*}\right)\right] \\
\dot{\hat{\Theta}}^{*} & =\bar{\gamma} \mu_{f}^{* T}\left(z_{1, f}+k_{2} z_{2, f}\right)-\bar{\gamma} \dot{\mu}_{f}^{* T} z_{2, f} \\
\beta_{\Theta}^{*} & =\bar{\gamma} \mu_{f}^{* T} z_{2, f}
\end{aligned}
$$

where $z_{1, f}=\left[z_{11, f}, z_{21, f}, z_{31, f}, z_{41, f}\right]^{T}, k_{2}=\operatorname{diag}\left\{k_{12}, k_{22}, k_{32}, k_{42}\right\}$, and $\bar{\gamma}>0$ is the adaptation gain with appropriate dimensions. Then, the boundedness of all the closed-loop signals is ensured and the tracking errors converge to zero asymptotically.

ProOF. Substituting (56) into (55) yields

$$
\left(\bar{g} S^{*}\right)^{-1} \dot{z}_{2, f}=-z_{1, f}-k_{2} z_{2, f}-\mu_{f} \zeta_{\Theta}^{*}
$$

where $\zeta_{\Theta}^{*}=\hat{\Theta}^{*}-\Theta^{*}+\beta_{\Theta}^{*}$ is the off-the-manifold. Then, note that (32),(59) and the calculation of the derivative of $\zeta_{\Theta}^{*}$ by using (57) and (58), it can be obtained that the conclusion of Theorem 2 holds by following a similar procedure of the proof of Theorem 1. The details are thus omitted here.

Remark 4. As in Theorem 2, algebraic loops are avoided since matrix $\left(1-Q^{*}\right)$ is strictly upper triangular, which implies that $v_{1}^{*}$ depends on $v_{2}^{*}, v_{3}^{*}$ and $v_{4}^{*}$; $v_{2}^{*}$ depends on $v_{3}^{*}$ and $v_{4}^{*}$; and $v_{3}^{*}$ only depends on $v_{4}^{*}$.

Remark 5. Except for actuator parameters, all other physical parameters such as mass, moment of inertia, etc. are not used in the controller design. Hence the proposed scheme is also robust to the uncertainties in the quadrotor body dynamics.

Remark 6. The controller (56)-(58) could be designed in an alternative way. Define the following filter,

$$
\dot{\mu}_{f}^{*}=-k_{f}\left(\mu_{f}^{*}-\mu^{*}\right)
$$

with $k_{f}>0$ the filter constant, then a modified version of the proposed control law is given by

$$
\begin{aligned}
v^{*} & =D^{*-1}\left[-z_{1}-k_{2} z_{2}-\mu_{f}\left(\hat{\Theta}^{*}+\beta_{\Theta}^{*}\right)\right] \\
\dot{\hat{\Theta}}^{*} & =\bar{\gamma} \mu_{f}^{* T}\left(z_{1}+k_{2} z_{2}\right)-\bar{\gamma} \dot{\mu}_{f}^{* T} z_{2} \\
\beta_{\Theta}^{*} & =\bar{\gamma} \mu_{f}^{* T} z_{2}
\end{aligned}
$$

From the above design, it is straightforward to conclude that if the filter parameter $k_{f}$ are chosen large enough such that $\mu_{f}^{*} \approx \mu^{*}$, one can ensure the stability of the closed-loop system by following a similar procedure mentioned above. Obviously, compared with the previous controller design, the modified one is easier to implement in practical and, the dynamical order of the whole system is reduced as well, at the cost of tracking accuracy (which depends on the value of the filter constant). 


\section{Simulation Results}

Simulation results are carried out in this section to verify the proposed controller. The (nominal) physical parameters of a "+"-type quadrotor used in simulation are borrowed from [5] as: $m=0.468 \mathrm{~kg}, g=9.81 \mathrm{~m} / \mathrm{s}^{2}, l=0.225 \mathrm{~m}$, $J_{x}=J_{y}=4.856 \times 10^{-3} \mathrm{~kg} \cdot \mathrm{m}^{2}, J_{z}=8.801 \times 10^{-3} \mathrm{~kg} \cdot \mathrm{m}^{2}, J_{r}=3.357 \times 10^{-5} \mathrm{~kg} \cdot \mathrm{m}^{2}, c_{t}=2.98 \times 10^{-6} \mathrm{~N} \cdot \mathrm{s}^{2} / \mathrm{rad}^{2}$ and $c_{d}=1.14 \times 10^{-7} \mathrm{Nm} \cdot \mathrm{s}^{2} / \mathrm{rad}^{2}$.

To verify the robustness of the designed controller, we consider the following different values of the time-varying actuator parameters: 1$)$ at the initial stage $(t=0 s)$, the thrust factors for the four rotors are set as $c_{t 1}=c_{t}, c_{t 2}=0.97 c_{t}$, $c_{t 3}=1.02 c_{t}$ and $c_{t 4}=0.98 c_{t}$; the drag factors are $c_{d 1}=c_{d}, c_{t 2}=1.02 c_{d}, c_{t 3}=0.96 c_{d}$ and $c_{t 4}=0.97 c_{d}$, and the motor-voltage gains are chosen as $K_{m 1}=100, K_{m 2}=97, K_{m 3}=95$ and $K_{m 4}=98$. 2) at time instant $t=60 \mathrm{~s}$, the motor of the 3rd rotor suffers from $30 \%$ LOE, i.e., $K_{m 3}=95$ is changed to $K_{m 3}=66.5$. 3) at time instant $t=90 \mathrm{~s}$, LOE is occured to the propeller system of the 4 th rotor, where $c_{t 4}$ and $c_{d 4}$ are lowered by $30 \%$. The controller parameters are chosen as: $k_{11}=k_{21}=k_{31}=20, k_{12}=k_{22}=k_{32}=5, k_{41}=0.5, k_{42}=2, k_{51}=k_{61}=1, k_{52}=k_{62}=0.6$ and $k_{0}=0.5$; the adaptation gain for each adaptation law for the attitude system is fixed at $1 \times 10^{-4}$, while for the altitude system is set to $1 \times 10^{-2}$.

At the beginning stage of this simulated example, the horizontal reference of the quadrotor is set as $x_{d}=y_{d}=$ $1-\exp (-t)$, and then it is changed to $x_{d}=\sin (0.05 \pi(t-30))+1$ and $y_{d}=\cos (0.05 \pi(t-30))$ at $t=30 \mathrm{~s}$. The altitude and yaw angle reference are fixed at $0.05 t$ and $0.1 \sin (0.1 \pi t)$, respectively.

For the sake of comparison, we also present a control scheme based on classical adaptive control method. For attitude and altitude subsystems, the adaptive controller is designed as

$$
\begin{aligned}
v_{C A}^{*} & =D^{*-1}\left(-z_{1}-k_{2} z_{2}-\mu^{*} \hat{\Theta}_{C A}^{*}\right) \\
\dot{\hat{\Theta}}_{C A}^{*} & =\gamma_{C A} \mu^{* T} z_{2}
\end{aligned}
$$

and the horizontal controller is the same with (46). All the control parameters of the classical adaptive controller are the same as the proposed one. In addition, the baseline controller is also considered in this simulation, where all the adaptation gains are set as zero. All the three controllers are tested under same conditions.

Simulation results are shown in Fig.2 and Fig.3, where the tracking errors of position and attitude are depicted respectively. From both figures we can see that, in the first $60 s$, the baseline controller exposes steady-state errors in the attitude and the height response, due to the effect of small uncertainties in the actuator parameters $c_{t}, c_{d}$ and $K_{m}$. However, the uncertainties are overcome by both adaptive controllers. In contrast, from Fig. 2, the horizontal response of the baseline controller performs similar to that of the adaptive controllers, because of the existence of the integral action in the control law. At $60 \mathrm{~s}$, where the first LOE occurs, it is shown that the baseline controller cannot adapt to the sudden change of the actuator parameter and become unstable. In the mean time, the system response of the two adaptive controllers are affected temporarily and then converge very fast, so is the case when another LOE occurring at $90 \mathrm{~s}$. Although both adaptive schemes can stay stable subject to the change of parameters, the I\&I adaptive one performs much better than that of the classical one, in terms of the transient behaviour, as shown in these figures.

\section{Experimental Results}

In this section, experimental results are carried out to illustrate the effectiveness of the proposed control scheme applied to an " $x$ "-type quadrotor setup, as shown in Fig.4. In this setup, the remote sends only the take off or landing commands to the quadrotor system, and is connected with the ground station via USB. The position and attitude data are sent to the remote through the wireless communication, and then these data can be transmitted to the ground station. The reference trajectories are already programmed in the system environment. In the experiments, we consider three cases: the first case exams the performance of the classical adaptive controller under nominal situation (i.e., no hardware or software faults occur) ; in the second case, the quadrotor is equipped with an intentionally damaged propeller in one of the rotors, and a comparison between the classical adaptive controller and the I\&I adaptive controller is given; the third case is implemented with the I\&I adaptive controller under the damaged propeller as the same as case 2, and LOE factors are injected to all of the four actuators.

\subsection{Case 1: Nominal Situation}

In this case, the experiment is carried out using the classical adaptive scheme (64). The purpose of this case is, to verify that the parameters of four actuators are actually distinct which are needed to be considered in the control 

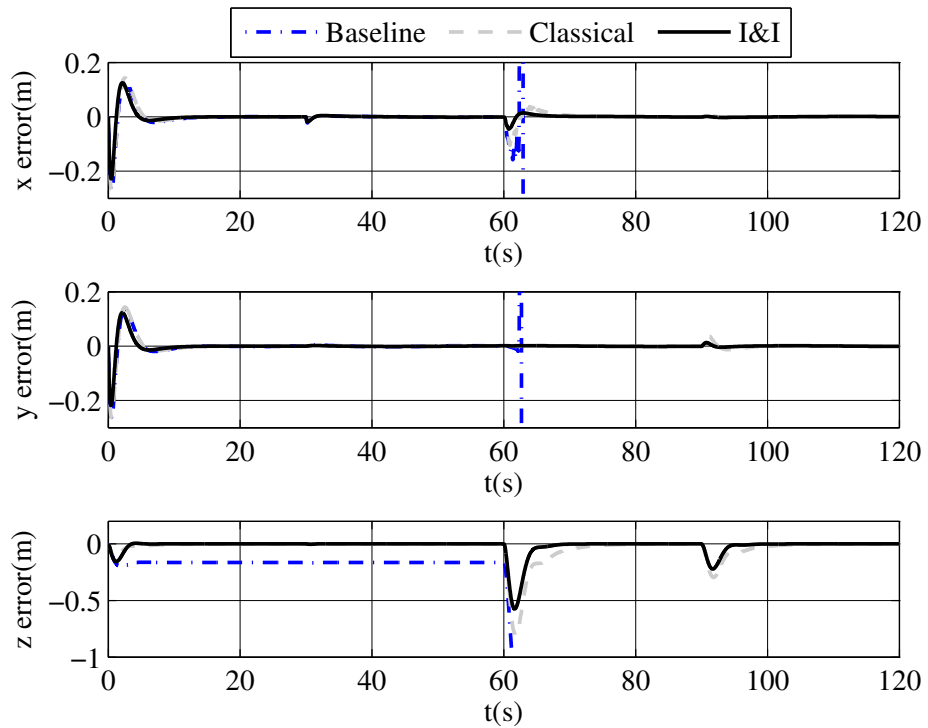

Figure 2: Simulation: the evolution of position errors of the three control schemes.
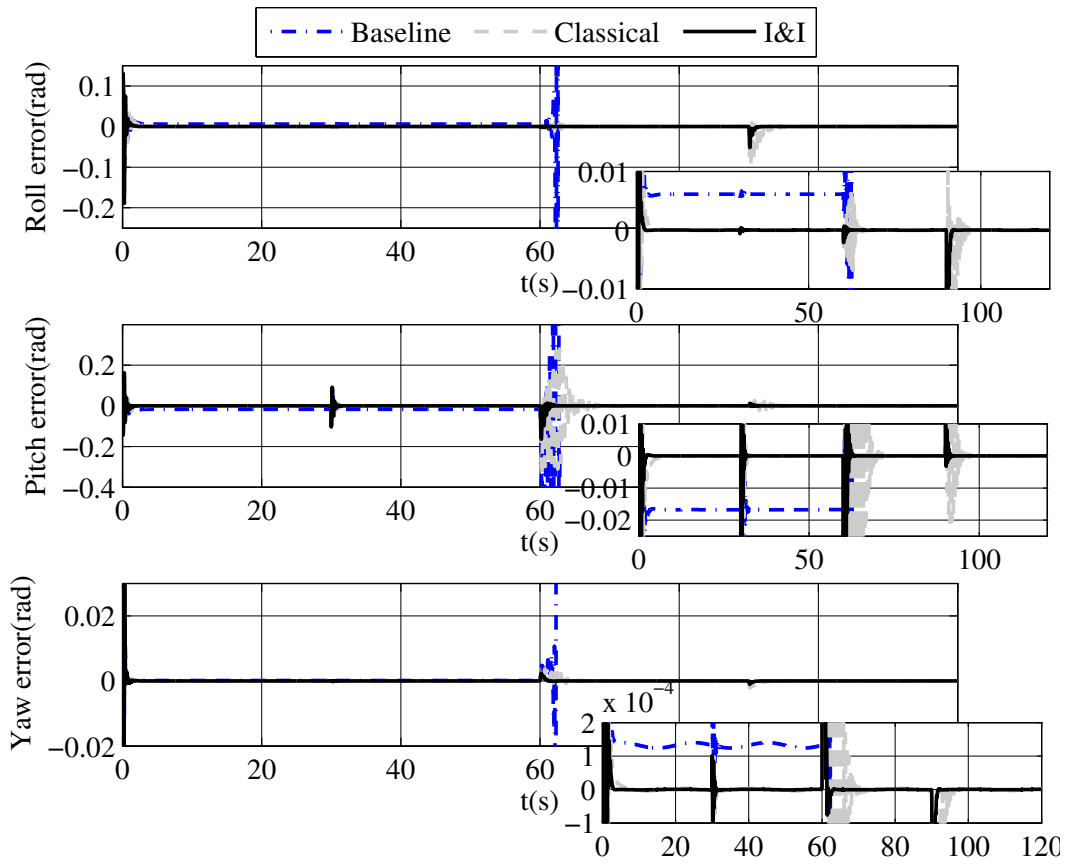

Figure 3: Simulation: the evolution of attitude errors of the three control schemes.

design, even no obvious changes or damages of the system are observed. To make a comparison, we also present experimental results with the baseline controller, whose adaption gains are all set as zero. The references in this case are $x_{d}=y_{d}=\psi_{d}=0$ and $z_{d}=0.6 /(1+\exp (-t+4))$.

Results of this case are shown in Fig.5 and Fig.6, where the curves of position and attitude errors are depicted respectively. As expected, the performances are improved by the adaptive controller, especially in the attitude responses. 


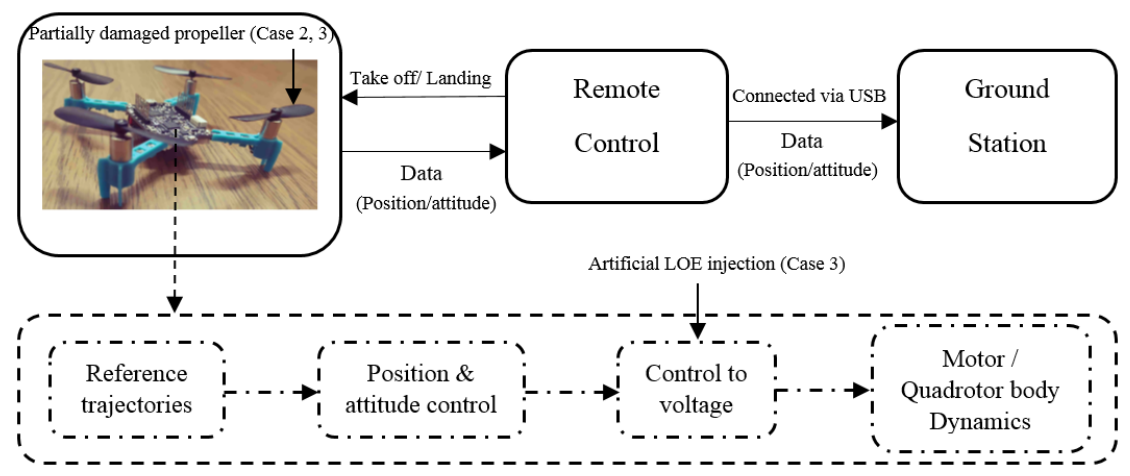

Figure 4: " $X$ "-type quadrotor platform.
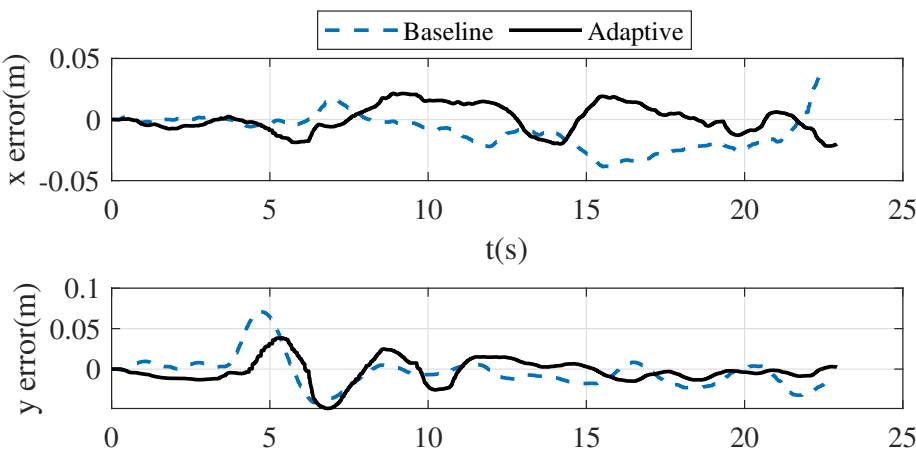

$\mathrm{t}(\mathrm{s})$

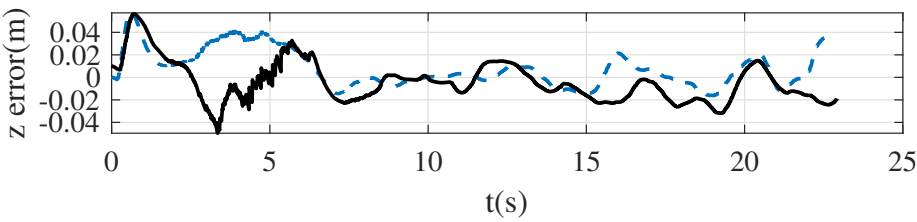

Figure 5: Case 1: the evolution of position error.

\subsection{Case 2: Performance Comparison}

In this case, to test the robustness of the system, the quadrotor platform is equipped with a partially damaged propeller of the 4th rotor, which makes the parameter of this rotor significantly different from all other ones. We are also interested in a further comparison of the classical and I\&I adaptive controllers experimentally. To implement the controller more easily, the adaptation gains of attitude and altitude subsystems are set as the same value. Different adaptation gains are set for the two controllers respectively. For the classical adaptive controller, the adaptation gains are chosen for three different values, $0.01,0.1$ and 1 , respectively, where $\gamma_{C A}=1$ is the max value can be chosen in the tuning process; and for the I\&I adaptive controller, the adaptation gains are all fixed at 0.01 , which is also the extreme one can be chosen in the experiment. The references in this case are: $x_{d}=0.7 \sin (0.05 \pi t), y_{d}=-0.7 \cos (0.05 \pi t)+0.7$, $z_{d}=0.05 t$ and $\psi_{d}=0$.

Experiment results are carried out using the above four controllers, namely, controller 1-4, as shown in Fig. 7 and Fig.8. Fig. 7 shows the trajectories in x-y plane and Fig. 8 presents the time profiles of the norms of the position error vector $e_{\xi}=\left[z_{41}, z_{51}, z_{61}\right]^{T}$ and the attitude error vector $e_{\Theta}=\left[z_{11}, z_{21}, z_{31}\right]^{T}$ respectively. In both figures, the improving performance are observed of the classical adaptive controller (controller 1-3) with the increasing of the adaptation gain. However, without larger adaptation gain, the I\&I adaptive one (controller 4) outperforms all other controllers. These figures illustrate the superiority of I\&I adaptive method in the practical situation. 


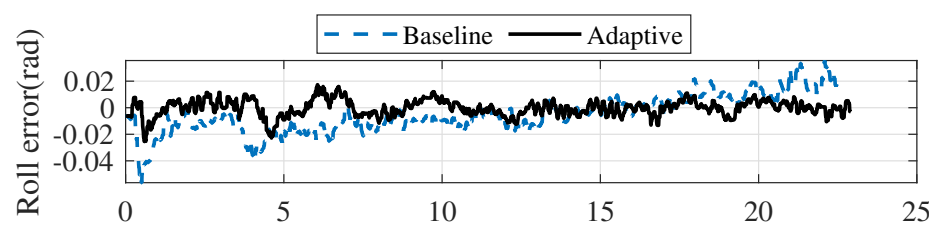

$\mathrm{t}(\mathrm{s})$

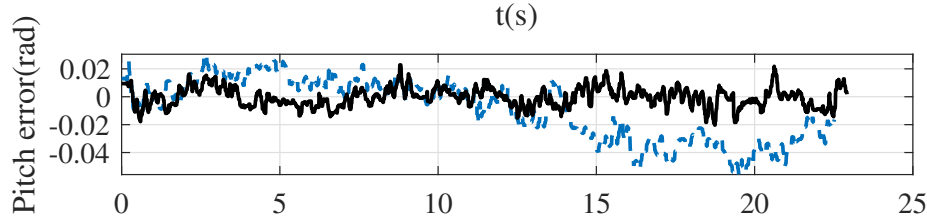

$\mathrm{t}(\mathrm{s})$



Figure 6: Case 1: the evolution of attitude error.

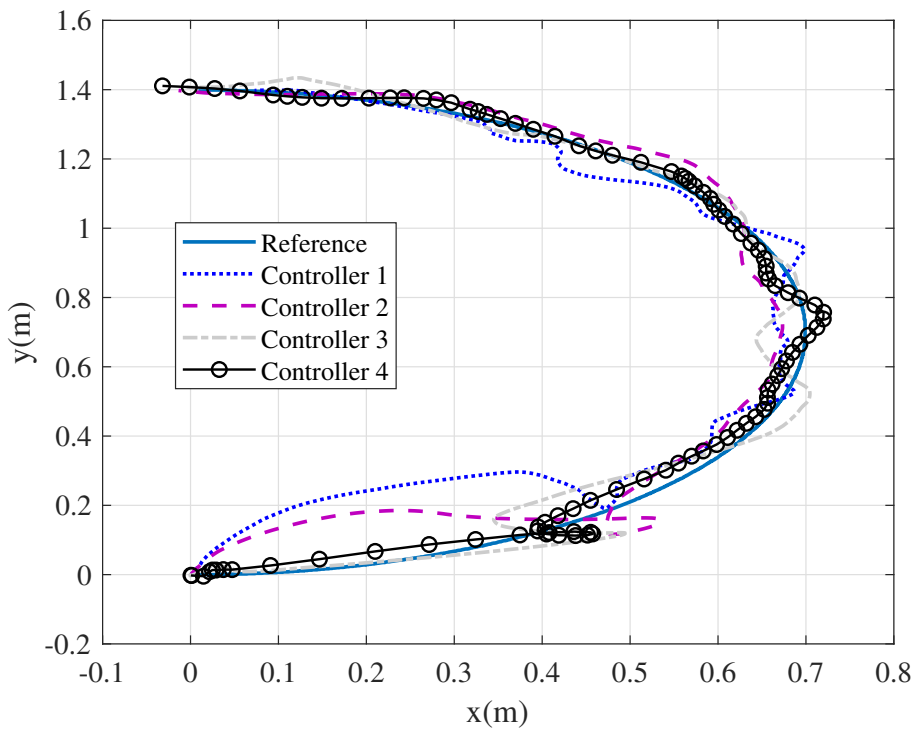

Figure 7: Case 2: the evolution of trajectory tracking in $x-y$ plane.

\subsection{Case 3: Software Fault Injection}

In this case, the LOE faults will be injected artificially in the system programming environment to test the robustness of the I\&I adaptive controller. This case is implemented on the basis of case 2, where the damaged propeller is still equipped. Initially, no LOEs occur in the first 20s; at 20s, the 1st rotor suffers from a $30 \%$ LOE; at 40 s, the 2 nd and the 3rd rotors are injected with $20 \%$ and $10 \%$ LOE, respectively; at 60 s, the $2 \mathrm{nd}$, 3rd and 4th rotors all suffer from $10 \%$ LOE; finally, at 80s, all faulty rotors return to healthy states, and remain unchanged in the rest of the flight. The quadrotor in this case is commanded to follow a circular trajectory in the horizontal direction, with the period of about $20 \mathrm{~s}$ and the magnitude of $0.7 \mathrm{~m}$. The height command is $1 \mathrm{~m}$ with a smooth transient stage and the yaw command is still zero. Results are shown in Fig. 9-11, where the system can track the reference constantly and the output errors 

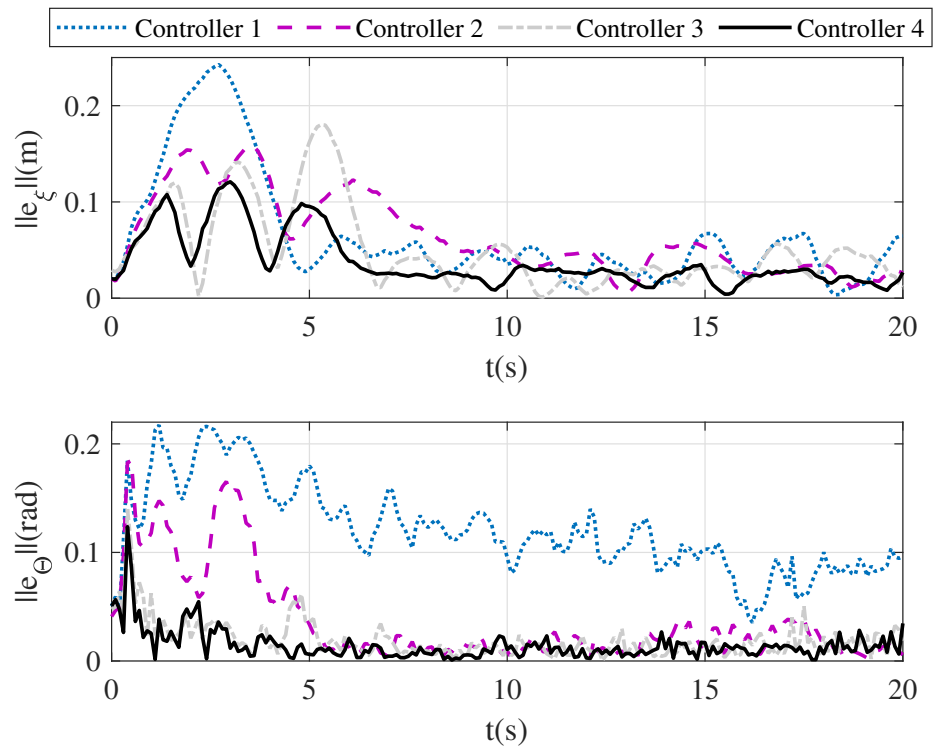

Figure 8: Case 2: the evolution of norms of position and attitude errors.

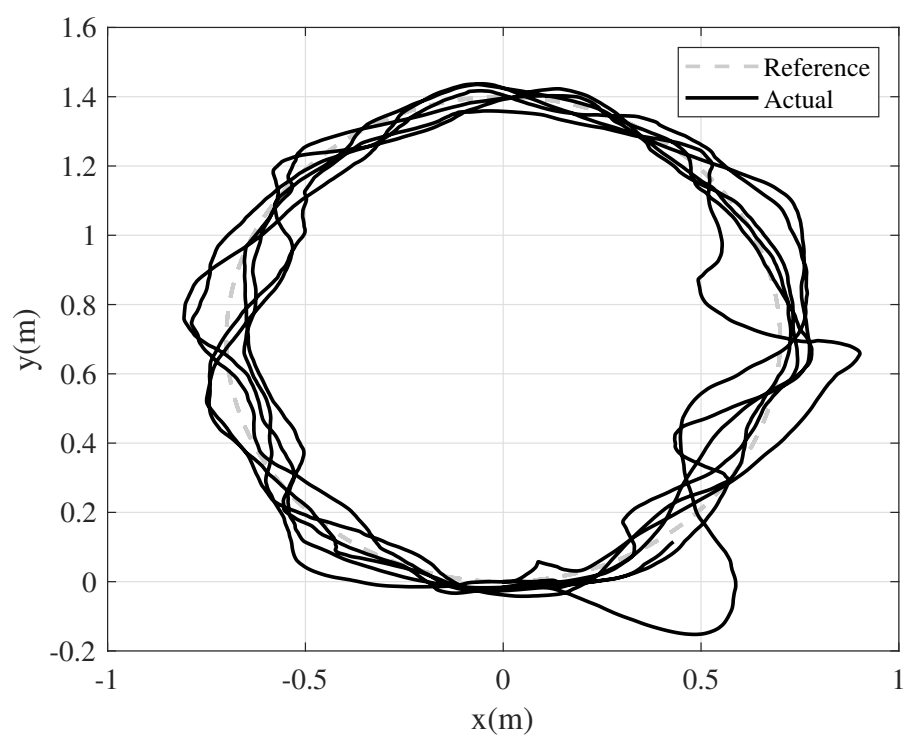

Figure 9: Case 3: the evolution of trajectory tracking in $x-y$ plane.

of the subsystems are kept within small ranges.

\section{Conclusion}

Two adaptive control schemes are proposed in this paper to cope with uncertain and faulty actuators for two of common configurations of quadrotors, namely, "+"-type and " $x$ "-type. By extracting unknown parameters from the original control inputs, actuator uncertainties are compensated via the constructed I\&I adaptive controller. To avoid the self-loop problem, we propose an enhanced control design which utilizes gain matrix decomposition technique. Simulation results show that the steady-state error and the PLOE of actuators can be efficiently handled. Finally, three 

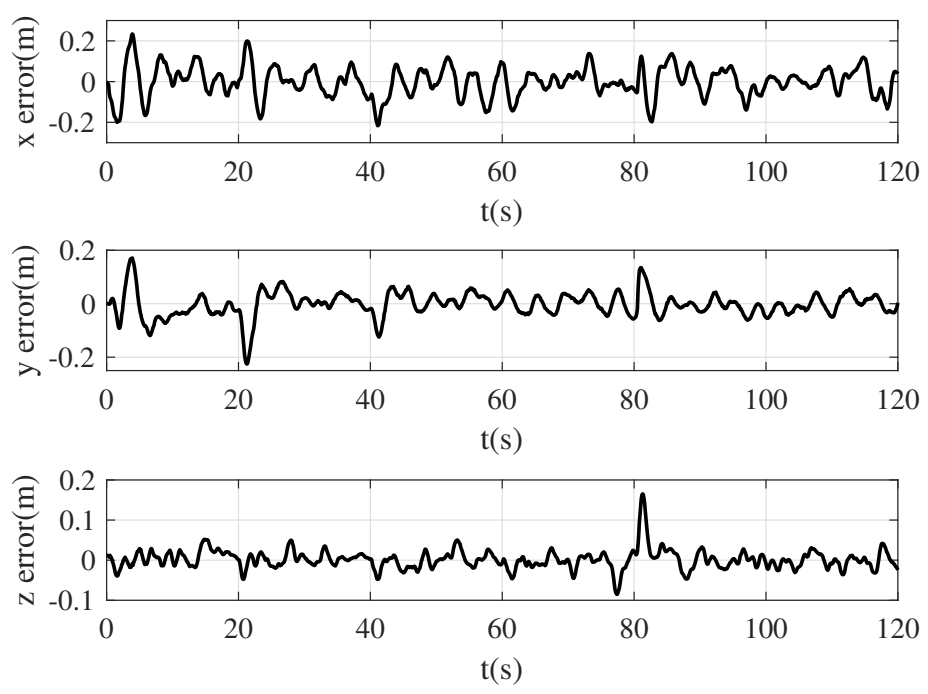

Figure 10: Case 3: the evolution of position error.
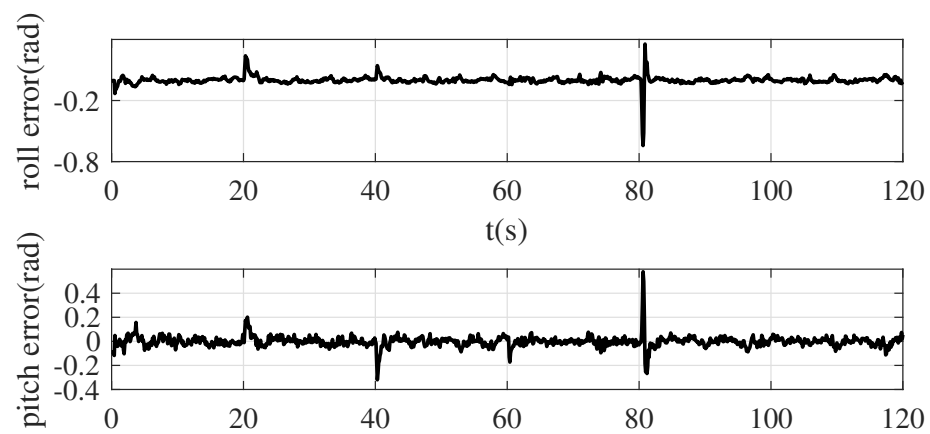

$\mathrm{t}(\mathrm{s})$

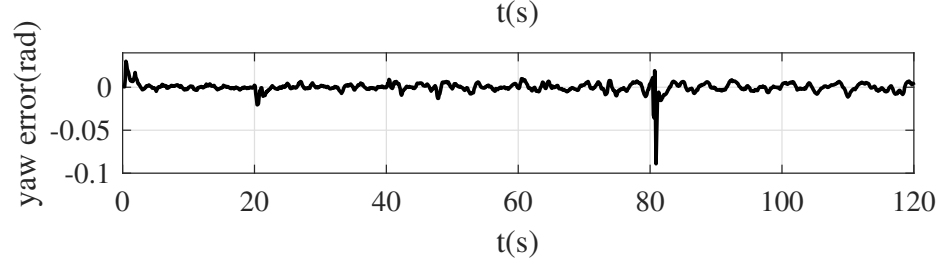

Figure 11: Case 3: the evolution of attitude error.

different scenarios of experimental results are carried out to show the superiority of the proposed controller.

Conflict of Interest: The authors declare that they have no conflict of interest.

Funding: This study was funded by National Natural Science Foundation of China, Grant/Award Number: 61673050.

Data availability statement: All data generated or analysed during this study are included in this published article (and its supplementary information files). The datasets generated during the current study are available from the 
corresponding author on reasonable request.

Qi Han received the B.E. degree in automation from Lanzhou Jiaotong University, Lanzhou, China, in 2018, and MS degree from Beijing Jiaotong University, China, in 2021. His interests includes robust adaptive control and formation control. E-mail: hanqi560@163.com

Xiangbin Liu received his BS degree from Xi' an Institute of Technology, China, in 1995, MS degree from University of Science and Technology Beijing, China, in 2002, and Ph.D. degree from Zhejiang University, China, in 2009, respectively. He is an associate professor at the School of Electronics and Information Engineering, Beijing Jiaotong University. His research interests include adaptive control, robust control and nonlinear control. E-mail: xbliu@bjtu.edu.cn

Zhitao Liu received his Ph.D. degree in control science and engineering from Zhejiang University, Hangzhou, China, in 2010. From 2011 to 2014, he was a Research Fellow with TUM CREATE, Singapore. Since 2017, he has been an Associate Professor with the Institute of Cyber-Systems and Control, Zhejiang University. His current research interests include robust adaptive control, wireless transfer systems, and energy management systems. Email:ztliu@zju.edu.cn

Hongye Su received his Ph.D. degree from Zhejiang University, China, in 1995. He has been promoted to the full Professor at the same university since 2000. Since 2014, he has held the positions of Director of the Institute of Cyber-Systems and Control, as well as Director of the State Key Laboratory of Industrial Control Technology at Zhejiang University. His main academic activities are in the areas of robust control, nonlinear adaptive control and chemical industrial control. E-mail: hysu@iipc.zju.edu.cn.

\section{References}

[1] X. Shao, N. Liu, Z. Wang, W. Zhang, W. Yang, Neuroadaptive integral robust control of visual quadrotor for tracking a moving object, Mech. Syst. Sig. Process. $136(2020) 106513$.

[2] T. Madani, A. Benallegue, Control of a quadrotor mini-helicopter via full state backstepping technique, in: Proc. IEEE Conf. Decision and Control, 2006, pp. 1515-1520.

[3] S. Bouabdallah, R. Siegwart, Backstepping and sliding mode techniques applied to an indoor micro quadrotor, in: Proc. IEEE Int. Conf. Robot. Autom., 2005, pp. 2247-2252.

[4] T. Madani, A. Benallegue, Backstepping control for a quadrotor helicopter, in: Proc. IEEE/RSJ Int. Conf. Intell. Robots Syst., 2006, pp. 32553260.

[5] A. Tayebi, S. Mcgilvray, Attitude stabilization of a four-rotor aerial robot, in: Proc. IEEE Conf. Decision and Control, 2004 , pp. 1216-1221.

[6] A. Tayebi, S. Mcgilvray, Attitude stabilization of a VTOL quadrotor aircraft, IEEE Trans. Control Syst. Technol. 14 (3) (2006) $562-571$.

[7] M. Huang, B. Xian, C. Diao, K. Yang, Y. Feng, Adaptive tracking control of underactuated quadrotor unmanned aerial vehicles via backstepping, in: Proc. Amer. Control Conf., 2010, pp. 2076-2081.

[8] G. Antonelli, E. Cataldi, F. Arrichiello, P. R. Giordano, S. Chiaverini, Adaptive trajectory tracking for quadrotor MAVs in presence of parameter uncertainties and external disturbances, IEEE Trans. Control Syst. Technol. 26 (1) (2018) 248-254.

[9] B. Zhao, B. Xian, Y. Zhang, X. Zhang, Nonlinear robust adaptive tracking control of a quadrotor UAV via immersion and invariance methodology, IEEE Trans. Ind. Electron. 62 (5) (2015) 2891-2902.

[10] B. Zhao, B. Xian, Y. Zhang, X. Zhang, Nonlinear robust sliding mode control of a quadrotor unmanned aerial vehicle based on immersion and invariance method, Int. J. Robust Nonlinear Control 25 (18) (2015) 3714-3731.

[11] Y. Zou, Z. Meng, Immersion and invariance-based adaptive controller for quadrotor systems, IEEE Trans. Syst., Man, Cybern. 49 (11) (2019) 2288-2297.

[12] W. Hao, B. Xian, Nonlinear adaptive fault-tolerant control for a quadrotor UAV based on immersion and invariance methodology, Nonlinear Dyn. 90 (2017) 2813-2826.

[13] P. Tang, F. Zhang, J. Ye, D. Lin, An integral TSMC-based adaptive fault-tolerant control for quadrotor with external disturbances and parametric uncertainties, Aerosp. Sci. Technol. 109 (2020) 106415.

[14] R. Avram, X. Zhang, J. Muse, Quadrotor actuator fault diagnosis and accommodation using nonlinear adaptive estimators, IEEE Trans. Control Syst. Technol. 25 (6) (2017) 2219-2226.

[15] R. Avram, X. Zhang, J. Muse, Nonlinear adaptive fault-tolerant quadrotor altitude and attitude tracking with multiple actuator faults, IEEE Trans. Control Syst. Technol. 26 (2) (2018) 701-707.

[16] J. Shi, Y. Yang, J. Sun, X. He, D. Zhou, Y. Zhong, Fault-tolerant formation control of non-Linear multi-Vehicle systems with application to quadrotors, IET Contr. Theory. Appl. 11 (17) (2017) 3179-3190. 
[17] F. Chen, F. Lu, B. Jiang, G. Tao, Adaptive compensation control of the quadrotor helicopter using quantum information technology and disturbance observer, J. Franklin Inst. 351 (1) (2014) 442-455.

[18] F. Chen, Q. Wu, B. Jiang, G. Tao, A reconfiguration scheme for quadrotor helicopter via simple adaptive control and quantum logic, IEEE Trans. Ind. Electron. 62 (7) (2015) 4328-4335.

[19] B. Wang, X. Yu, L. Mu, Y. Zhang, Disturbance observer-based adaptive fault-tolerant control for a quadrotor helicopter subject to parametric uncertainties and external disturbances, Mech. Syst. Sig. Process. 120 (2019) 727-743.

[20] M. Amoozgar, A. Chamseddine, Y. Zhang, Fault-tolerant fuzzy gain-scheduled PID for a quadrotor helicopter testbed in the presence of actuator faults, in: IFAC Proc. Vol. 45 (3) (2012) 282-287.

[21] Y. Song, L. He, D. Zhang, et al, Neuroadaptive fault-tolerant control of quadrotor UAVs: a more affordable solution, IEEE Trans. Neural Netw. Learn. Syst. 30 (7) (2019) 1975-1983.

[22] A. Merheb, H. Noura, F. Bateman, Emergency control of AR drone quadrotor UAV suffering a total loss of one rotor, IEEE/ASME Trans. Mechatronics 22 (2) (2017) 961-971.

[23] A. Astolfi, D. Karagiannis, R. Ortega, Nonlinear and adaptive control with applications, Springer: London, 2008.

[24] D. Seo, M. Akella, Non-certainty equivalent adaptive control for robot manipulator systems, Syst. Control Lett. 58 (4) (2009) $304-308$.

[25] D. Seo, M Akella, High-performance spacecraft adaptive attitude-tracking control through attracting-manifold design, J. Guid. Control Dynam. 31 (4) (2008) 884-891.

[26] R. Costa, L. Hsu, A Imai, P. Kokotovic, Lyapunov-based adaptive control of MIMO systems, Automatica 39 (7) (2003) $1251-1257$.

[27] J. Chen, A. Behal, D. Dawson, Adaptive output feedback control for a class of MIMO nonlinear systems, in: Proc. Amer. Control Conf., 2006, pp. 5300-5305.

[28] C. Wang, C. Wen, X. Zhang, J. Huang, Output-feedback adaptive control for a class of MIMO nonlinear systems with actuator and sensor faults, J. Franklin Inst. 357 (12) (2020),7962-7982. 